\title{
LIMIT STATE ANALYSIS OF EARTHEN SLOPES USING DUAL CONTINUUM/FEM APPROACHES
}

\author{
COLBY C. SWAN, ${ }^{1 *}$ YOUNG-KYO SEO ${ }^{2}$ \\ ${ }^{1}$ Department of Civil and Environmental Engineering, The University of Iowa, 2404 Engineering Building, \\ Iowa City, IA 52242, U.S.A. \\ ${ }^{2}$ Computational Solid Mechanics Laboratory, Department of Civil and Environmental Engineering, \\ The University of Iowa, Iowa City, IA, 52242, U.S.A.
}

\begin{abstract}
SUMMARY
A framework alternative to that of classical slope stability analysis is developed, wherein the soil mass is treated as a continuum and in-situ soil stresses and strengths are computed accurately using inelastic finite element methods with general constitutive models. Within this framework, two alternative methods of stability analysis are presented. In the first, the strength characteristics of the soil mass are held constant, and the gravitational loading on the slope system is increased until failure is initiated by well-defined mechanisms. In the second approach, the gravity loading on the slope system is held constant, while the strength parameters of the soil mass are gradually decreased until well-defined failure mechanisms develop. Details on applying both of the proposed methods, and comparisons of their characteristics on a number of solved example problems are presented. Copyright (C) 1999 John Wiley \& Sons, Ltd.
\end{abstract}

KEY WORDS: slope failure; slope stability analysis; finite element method; soil plasticity models; limit state analysis

\section{INTRODUCTION AND MOTIVATION}

The objective of this paper is to briefly develop and compare the characteristics of two complementary approaches to earthen slope stability analysis which are amenable to usage in a general purpose Finite Element Method (FEM) framework. A fuller comparison and exposition of the two methods can be found in Reference. ${ }^{1}$ The essence of the first method is based on monotonically increasing the gravity loading on soil slopes of fixed strength characteristics until well-defined failure mechanisms develop, while the essence of the second approach involves holding the gravity loading on slope systems constant, while reducing the strength characteristics of the soil mass until limit state mechanisms develop. Once the basic methods are developed, their basic characteristics are explored through a number of slope stability test problems. Both methods appear to be more general and robust than classical slip-surface-type slope stability analysis techniques which generally assume classical Mohr-Coulomb soil behaviours.

In classical Slope Stability Analysis (SSA), a sloped soil mass is subjected to realistic self-weight loading and the objective is to find the continuous surface passing through the soil mass, which has the minimum Factor of Safety (FS) against sliding or shear failure. It is noted that computation of factors of safety against failure in accordance with this approach can be

*Correspondence to: Colby C. Swan, Department of Civil and Environmental Engineering, The University of Iowa, 2404 Engineering Building, Iowa City, IA 52242, U.S.A. E-mail: swan@diego.ecn.uiowa.edu

CCC 0363-9061/99/121359-13\$17.50

Received 4 November 1998

Copyright (C) 1999 John Wiley \& Sons, Ltd.

Revised 14 January 1999 
a challenging proposition even for homogeneous soil deposits in that it involves in situ computation of soil stresses, and shear strengths which are generally dependent upon the in situ stress. Furthermore, it is well established that the search for the continuous critical surface $\Gamma^{*}$ which has the minimal FS is nontrivial. ${ }^{2}$ A variety of approximate and specialized methods dating from the late 19th to mid 20th century exist for computing for computing global factors of safety depending upon the slope configuration height, and steepness, as well as the constitutive properties of the soil which comprise the slope. A thorough review of many of these classical methods of slope stability analysis methods has been provided by Nash. ${ }^{3}$ The limitations of many of these classical approaches are that Mohr-Coulomb shear strength behaviour with a fixed friction angle is typically assumed, and that the computation of stresses and dependent shear strengths in the soil mass is approximate due to application of simple statistics methods to inherently statically indeterminate continuum mechanics problems. To reduce the manual trial and error associated with these techniques, as with slice methods, a number of engineering software packages have been developed, but most still compute soil stresses and strengths approximately using variations of simple statics methods.

In recent years, finite element methods have been increasingly used to predict both displacements and stresses in statically indeterminate slopes, dams and embankments. ${ }^{4}$ The potentially attractive aspects of computing slope stabilities within a continuum/FEM framework are many: (1) the equilibrium stresses, strains, and the associated shear strengths in the soil mass can be computed very accurately; (2) general soil material models (including Mohr-Coulomb and numerous others) can be employed; (3) the method can be applied with complex slope cofigurations and soil deposits in two or three dimensions to model virtually all types of mechanisms; ${ }^{5}$ (4) the critical failure mechanism developed can be extremely general, and need not be simple circular or logarithmic spiral arcs; and (5) the method can be extended to account for seepageinduced failures, brittle soil behaviours, random field soil properties, and engineering interventions such as geo-textiles, soil nailing, drains and retaining walls.

When using finite element methods for slope stability analysis, however, there are a number of possibilities. One approach demonstrated by Huang ${ }^{6}$ used elastic FEM analysis to compute the stress field in an earthen dam with steady-state seepage. Based on computed stress field and pore-pressure fields, which were assumed to be de-coupled, contours of the local factor safety against shear failure with respect to a general criterion were then computed and displayed. Interestingly, the results of such analysis by Huang ${ }^{6}$ demonstrated that contours of minimal local factors of safety against shear failure do not necessarily constitute potential failure mechanisms since they are often fully contained within the soil mass. Since contours of minimal local factors of safety against shear failure do not necessarily represent plausible slope or embankment failure mechanisms, it is often necessary to use inelastic finite element methods to fully initiate and find the critical failure mechanisms and their associated factors of safety.

Using inelastic computational methods to perform slope stability analysis, two complementary techniques have been employed to date:

1. a slope model of fixed soil shear strengths is utilized, and gravity loading on the model is gradually increased until critical failure mechanisms develop; ${ }^{7}$ and

2. fixed gravity loading is applied to a slope model, and the shear strengths of the comprising soils are gradually reduced until critical failure mechanisms develop. ${ }^{8,9}$

The first approach (or the gravity loading to failure method) has been demonstrated previously by Chen and Mizuno ${ }^{7}$ for the case of cohesive soils but not for the case of strictly frictional soils. 
The second approach (or the strength-reducing method) has recently been applied by Griffiths and Kidger $^{8}$ for slopes of homogeneous, purely cohesive soil modelled with von Mises elastoplasticity. Earlier, Zienkiewicz et al. ${ }^{9}$ employed similar methods using viscoplastic Mohr-Coulomb soil models. Since the preceding works involved manual trial and error on the part of the analyst, in this work, algorithms for automated limit state analysis are implemented. In addition, the comparative validity and efficacy of the proposed methods is examined in the context of specific examples.

Since the intent of the present work is to establish a basic framework for computing the initiation of slope stabilities, and not necessarily the accurate modeling of post-initiation behaviours, a small-deformation continuum formulation with non-softening elasto-plastic material models is utilized here. The modelling of post-stability behaviours by including large deformation, material softening, and contact effects are issues that can be addressed in future extensions of this framework. The methods being investigated here can be applied for both soils treated as a single-phase continuum, or soils treated as a multi-phase fluid-solid continuum. To highlight the essentials of the methods, attention is confined here to treatment of the soil as a single-phase continuum. The case of embankment failures with pore-pressure diffusion effects taken into account is treated as an extension of this work in Reference 10 and the treatment of slope failures under activity steady-state unconfined seepage is treated in Reference. ${ }^{1}$

\section{DUAL METHODS OF SLOPE STABILITY ANALYSIS METHOD}

\subsection{The basic analysis problem}

In the following, the response of soil slopes subjected to gravitational loading will be treated as general materially non-linear elliptic boundary value problems. To demonstrate the essential features of the method, the soil mass is here treated as either 'fully drained' with no seepage forces, or 'fully undrained.' In both treatments we will, for simplicity, be concerned only with total stresses and skeletal displacements, and not with pore pressure and seepage effects. (In other related works, pore pressure effects and seepage are explicitly taken into account. ${ }^{1,10}$ With seepage and pore pressure effects assumed negligible, and the additional assumption of inviscid soil constitutive models, the problems to be solved here feature no physical time dependence. Therefore, quasi-static elliptic boundary value problems will be solved to analyse the stability of the slope. Any and all time dependence in the problems being solved is simply for the application of time-based parametric loads.

Starting with continuum equilibrium, standard variational arguments can be applied to derive non-linear finite element equations governing the equilibrium of the soil mass as at the $A$ th node and $(n+1)$ th time step:

$$
\left(\mathbf{r}_{A}\right)_{n+1}=\left(\mathbf{f}_{A}^{\mathrm{int}}\right)_{n+1}-\left(\mathbf{f}_{A}^{\mathrm{ext}}\right)_{n+1}=0
$$

where

$$
\begin{gathered}
\left(\mathbf{f}_{A}^{\mathrm{int}}\right)_{n+1}=\int_{s} \mathbf{B}_{A}^{\mathrm{T}}: \boldsymbol{\sigma}_{n+1} \mathrm{~d} \Omega_{S} \\
\left(\mathbf{f}_{A}^{\mathrm{ext}}\right)_{n+1}=\int_{S} \rho N_{A} \mathbf{g}_{n+1} \mathrm{~d} \Omega_{S}+\int_{\Gamma_{h}} \mathbf{N}_{A} \mathbf{h}_{n+1} \mathrm{~d} \Gamma_{h}
\end{gathered}
$$


In general, equation (1) represents a set of non-linear algebraic equilibrium equations which must be solved in an iterative fashion for the incremental displacement field $(\Delta \mathbf{u})_{n+1}=\mathbf{u}_{n+1}-\mathbf{u}_{n}$ for each time step of the analysis problem. Numerous options exist for solving non-linear systems such as equations (1) and (2), a few of which are reviewed in detail in Reference 11 but here a Newton's method with line searching is used. ${ }^{12}$

In equation (2), $\mathbf{B}_{A}$ represents the nodal strain displacement matrix $\left(\mathbf{B}_{A}=\nabla^{s} N_{A}(\mathbf{x})\right)$, and $N_{A}$ denotes the nodal basis function for the $A$ th node. The quantity $\left(\mathbf{f}_{A}^{\text {int }}\right)_{n+1}$ represents the internal forces on node $A$ at time $t_{n+1}$ due to stresses in the soil mass, and $\left(\mathbf{f}_{A}^{\text {ext }}\right)_{n+1}$ represents the external forces applied to node $A$ at time $t_{n+1}$ due to body force and traction-type loads. As long as balance can be achieved between the internal soil stresses and external forces, then the slope will be stable with respect to the applied loads, and solutions to equation (1) will exist. When this balance can no longer be achieved, however, due to finite soil strength and increased gravity loading, then the slope will become unstable and on the verge of failure, since equilibrium solutions satisfying equation (1) will not exist.

\subsection{Limit analysis via gravity increase method}

For the gravity-induced method of slope stability analysis problems, the objective is to steadily increase the gravitational loading applied to the soil mass until the slope becomes unstable, and equilibrium solutions satisfying equation (1) can no longer be obtained. By monotonically increasing the magnitude of $\mathbf{g}(t)$, the magnitude of applied external forces $\mathbf{f}^{\text {ext }}$ [equation (2b)] increase until the soil mass reaches the limit of its resistive capacity (or strength) and is on the verge of unstable failure.

To avoid unnecessary manual intervention in the limit state analysis problem, the solution algorithm of Figure 1 is employed since it reliably and automatically finds the limit state (assuming it exists). ${ }^{\dagger}$ In the proposed gravity stability analysis method, the gravitational vector is prescribed as a function of time as follows:

$$
\mathbf{g}(t)=\dot{\mathbf{g}} \cdot t
$$

where $\dot{\mathbf{g}}$ is a prescribed vector specifying the direction of gravity loading and its rate of increase with time, and $t$ is a parametric time variable. By prescribing the applied gravitational acceleration vector $\mathbf{g}(t)$ in this manner, the limit analysis problem reduces simply to finding the largest time $t=t_{\text {limit }}$ for which a global equilibrium solution of equation (1) exists, and the limiting gravitational acceleration on the system is then merely

$$
\mathbf{g}_{\text {limit }}=\dot{\mathbf{g}} \cdot t_{\text {limit }}
$$

The algorithm of Figure 1 is merely one in which the time $t_{\text {limit }}$, which is not known $a$ priori, is approached asymptotically. For values of $t>t_{\text {limit }}$ solution of equation (1) will not exist. Thus if during the $(n+1)$ th time step the robust Newton's Method with line-searching fails to find a solution of equation (1), it is reasonably assumed that a solution does not exist and that $t_{n+1}>t_{\text {limit }}$. In this case, the time step $\Delta t$ is quartered and the algorithm reverts to time $t_{n}$ which is the most recent (or largest) time for which it is known that a solution to equation (1) exists. In the

\footnotetext{
${ }^{\dagger}$ For linear elastic soils, limit states will clearly not exist. For soils with finite shear strengths, limits tates will typically exist. Further discussion on existence of limit states is presented in Section 4.
} 


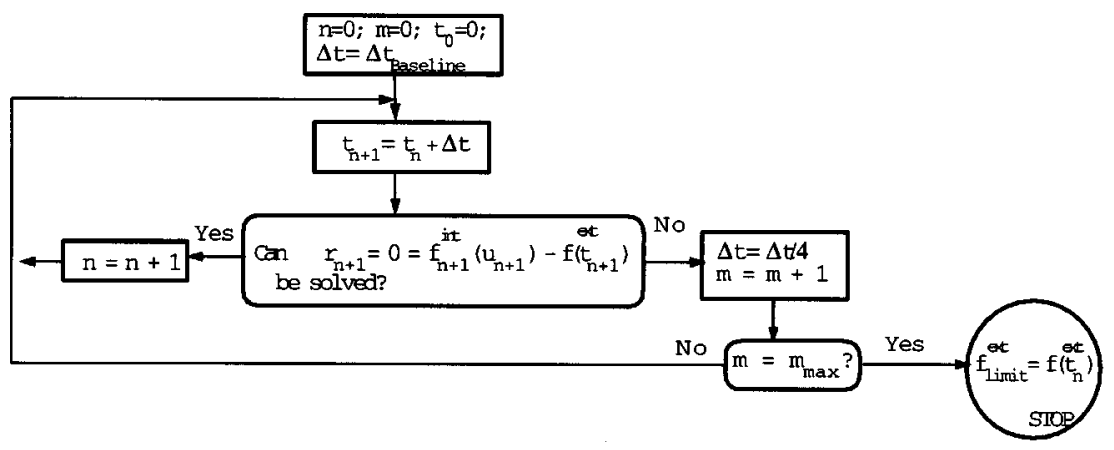

Figure 1. Automated limit state analysis algorithm

algorithm of Figure 1, the authors typically set the parameter $m_{\max }$ between 10 and 20, with larger values leading to more precise calculations of $t_{\text {limit }}$, and hence $\mathbf{g}_{\text {limit }}$.

In accordance with the fact that this method uses gravitational loading to induce slope failures, the proposed gravity-based factor of safety against slope failure is simply

$$
(\mathrm{FS})_{\mathrm{gi}}=\frac{g_{\text {limit }}}{g_{\text {actual }}}
$$

in which $g_{\text {actual }}$ is an appropriate and representative actual gravitational acceleration for the slope being analysed (i.e. $g_{\text {actual }} \simeq 9 \cdot 81 \mathrm{~m} \mathrm{~s}^{-2}=32 \cdot 2 \mathrm{ft} \mathrm{s}^{-2}$ ). Clearly, the higher the computed factor of safety, the more stable a slope is against failure, with values less than unity indicating that a given slope is unstable under actual gravity loading.

\subsection{Limit analysis via the strength reduction method}

With the strength reduction method of slope stability analysis, the basic form of the continuum equilibrium problem and the corresponding finite element equilibrium problem for each time step of analysis are essentially the same as for the gravity loading method of slope stability analysis. Accordingly, in the strength-reduction method, the algorithm used to solve the resulting system of non-linear finite element equations is also Newton's Method with line searching. The primary difference that comes about with the strength-reducing method is that with time, the shear strength parameters of the soil mass are monotonically decreased. The shear strength parameters of the soil mass can be reduced quite easily by utilizing a monotonically decreasing time function to govern the shear strength properties of the soil mass. Thus a typical shear strength parameter $Y$ for the soil mass could be governed in time as follows:

$$
Y(t)=Y_{\text {baseline }} * f(t)
$$

where $Y_{\text {baseline }}$ represents the estimated actual soil strength. For such a problem, actual gravity loading is applied to the soil mass at the outset of the problem, and the strength values of the soils comprising the slope, which are initially much higher than their true values, are monotonically reduced until solutions of the equilibrium problem (1) can no longer be found. As with the gravity loading method of stability analysis, the objective is find the maximum $t=t_{\text {limit }}$ for which 
a solution exists using the algorithm of Figure 1. Once this value is found, the computed factor of safety for the slope system for the strength reduction method is simply

$$
(\mathrm{FS})_{\mathrm{sr}}=\frac{Y_{\text {baseline }}}{Y(t)_{\text {limit }}}=\frac{1}{f\left(t_{\text {limit }}\right)}
$$

\section{A NONLINEAR DRUCKER-PRAGER ELASTO-PLASTICITY MODEL}

\subsection{Model description}

While the proposed slope stability analysis methods described above can be applied with a wide variety of soil plasticity models, they will generally converge more rapidly and produce more meaningful results when used with realistic soil models that feature continuous and smoothly differentiable rate constitutive equations. To efficiently and realistically model coupling between tensile, compressive, and shearing modes of ductile soil plasticity, a non-linear Drucker-Prager model with saturating incremental frictional effects (Figure 2) is employed in the calculations that follow in Section 4. In this section, the basic constitutive equations for this relatively simply soil model are briefly discussed.

Utilizing the assumption of small deformations, the strain tensor admits the additive elastic-plastic decomposition

$$
\varepsilon=\varepsilon^{\mathrm{e}}+\varepsilon^{\mathrm{p}}
$$

where $\varepsilon, \varepsilon^{\mathrm{e}}, \varepsilon^{\mathrm{p}}$ are, respectively, the total, elastic, and plastic strain tensors. The small deformation incremental stress response of the soil is assumed to be related to the strain response by

$$
\dot{\boldsymbol{\sigma}}=\mathbf{C}:\left(\dot{\varepsilon}-\dot{\mathrm{e}}^{\mathrm{p}}\right)
$$

where $\mathbf{C}$ is a fourth-order isotropic tensor of elastic moduli $\mathbf{C}=K \mathbf{1} \otimes \mathbf{1}+2 \mu \mathbf{I}_{\mathrm{dev}}$, in which $K$ is the bulk modulus of the soil and $\mu$ is the shear modulus. Using standard continuum mechanics notation, tensile stresses are taken as positive, and compressive stresses as negative.

In stress space, the elastic domain is bounded by a single non-hardening yield surface whose mathematical form is

$$
f(\boldsymbol{\sigma})=\|\mathbf{s}\|-\left\{\alpha+\lambda\left(1-\exp \left[\beta I_{1}\right]\right)\right\}=0
$$

where $\mathbf{s}$ is the deviatoric stress tensor and $\|\mathbf{s}\|^{2}=\frac{1}{2} J_{2} ; I_{1} \equiv \operatorname{tr}(\boldsymbol{\sigma})$ is the first invariant of the stress tensor and is negative in compression and positive in tension; and $\alpha, \lambda$, and $\beta$ are soil shearstrength parameters. For large compressive confining stresses $\left(I_{1} \rightarrow-\infty\right)$ the shear $f(\boldsymbol{\sigma})=\alpha+\lambda$ and is thus constant, thus leading to the preceding statement that this model features saturation of incremental frictional effect. Loading and unloading criteria for this plasticity model are specified by the Karesh-Kuhn-Tucker conditions

$$
f \leqslant 0, \quad \dot{\gamma} \geqslant 0, \quad \dot{\gamma} f=0
$$

with plastic consistency expressed by $\dot{\gamma} \dot{f}=0$. The flow rule for this model is associated, and thus

$$
\dot{\varepsilon}^{\mathrm{p}}=\dot{\gamma} \frac{\partial f}{\partial \sigma}
$$

This soil plasticity model is employed for the calculations of Section 4 using a fully implicit Backward Euler integration algorithm along with consistent tangent operators. ${ }^{13}$ 


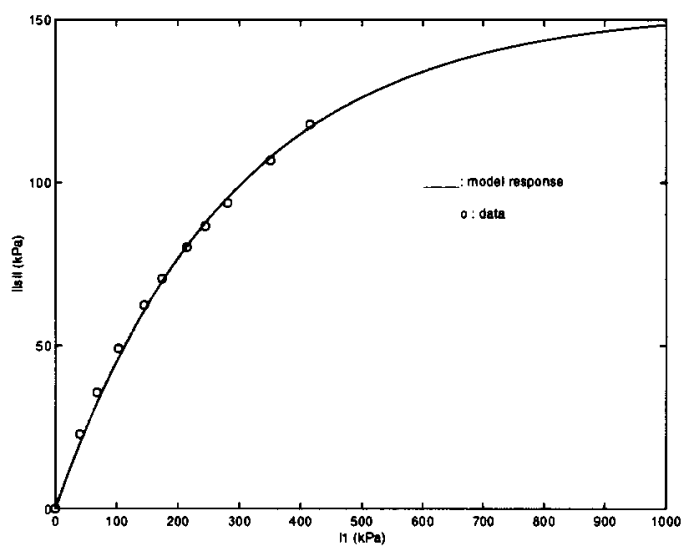

Figure 2. Yield surface of Drucker-Prager plasticity model employed with data used to select parameters for a sandy soil

The Mohr-Coulomb yield criterion has a long history of usage in classical soil shear strength characteristics in terms of the Mohr-Coulomb cohesion $c$ and friction and $\phi$. Here, the non-linear Drucker-Prager failure envelope with saturating frictional effects is employed for two reasons:

1. It is more realistic in that it predicts a saturation of soil strength with increasing effective confining stresses. The classical Mohr-Coulomb model unrealistically predicts no saturation of shear strength with increasing effective normal confining stresses.

2. The Drucker-Prager model does not suffer from the non-smooth corner regions that generally afflict Mohr-Coulomb-type soil models. While implementations of MohrCoulomb models have been developed wherein corners in the yield surface are smoothed, for computational implementation and numerical performance reasons, a number of investigators prefer Drucker-Prager and cap model-type plasticity models due to the relative smoothness of the yield surface, the corresponding lack of sharp corners, the ability to model ductile tensile failure, and the coupling between shear failure compressive plasticity.

So that the results of the present work can be compared with results of classical slope-stability analysis methods wherein the Mohr-Coulomb failure criterion is routinely considered, the yield criterion in equation (10) is re-written by taking its Taylor series expansion about $I_{1}=0$ :

$$
f(\boldsymbol{\sigma})=\|\mathbf{s}\|-\left\{\alpha-\theta I_{1}\left[1+\frac{\beta I}{2}+\frac{\left(\beta I_{1}\right)^{2}}{6}+\frac{\left(\beta I_{1}\right)^{3}}{24}+\cdots\right]\right\}
$$

where $\theta \doteq \lambda \beta$ is the slope of the envelope at $I_{1}=0$. For small values of $I_{1}$ (i.e. $\beta I_{1} \ll 1$ ), the linearized form of the yield function is

$$
f(\boldsymbol{\sigma})=\|\mathbf{s}\|-\left\{\alpha-\theta I_{1}\right\}
$$

which is simply a variation of linear Ducker-Prager yield criterion. A correspondence can be established between the two parameters $\alpha$ and $\theta$ of the linearized Drucker-Prager envelope (14) and the two parameters $c$ and $\phi$ of the Mohr-Coulomb envelope. For example, translations from 
Mohr-Coulomb parameters to linear Drucker-Prager parameters have been provided in Reference $^{14}$ as

$$
\alpha=\frac{\sqrt{2} c}{\left(1+4 / 3 \tan ^{2} \phi\right)^{1 / 2}} \quad \theta=\frac{\sqrt{2} \tan \phi}{3\left(1+4 / 3 \tan ^{2} \phi\right)^{1 / 2}}
$$

These equations can be inverted to provided a translation from linear Drucker-Prager envelope parameters to Mohr-Coulomb which will be used in the following section

$$
c=\frac{\alpha}{\sqrt{2}}\left(1+\frac{4}{3} \tan ^{2} \phi\right)^{1 / 2}, \quad \tan \phi=\frac{3}{\sqrt{2}} \theta\left(1-6 \theta^{2}\right)^{-1 / 2}
$$

\subsection{Soil model parameters}

So, that the demonstrative slope stability computations of the following section will be fairly realistic, parameters for the soil plasticity model are chosen carefully. The example computations of the next section are performed with two soil types: a cohesive, undrained clay, and a cohesionless frictional sand. The Drucker-Prager material model parameters for these soil types are listed in Table I. It is worth noting here that the shear strength behaviour for the sand differs quite substantially from that predicted using a classical Mohr-Coulomb model. Experimental measurements of sand shear strength properties presented in Reference 15 were used to select the model parameters $\lambda$ and $\beta$. The agreement between the model response and the published experimental soil response is shown in Figure 2.

\section{COMPARATIVE EXAMPLES}

In the examples that follow, the intention is to compare the relative performance characteristics of the gravity and strength-reduction methods of slope stability analysis. The example problems include soil slopes having both purely cohesive soils, purely frictional soils, and heterogeneous soils.

\subsection{Non-frictional, purely cohesive soils}

Both the strength reduction and gravity methods of slope stability analysis are applied here to a sloped soil mass of uniform cohesive clay soil properties listed in Table I, and having a height of

Table I. Clay and sand materials parameters used in slope stability computations

\begin{tabular}{ccc}
\hline Material parameter & Clay values & Sandy values \\
\hline$\rho$ & $1800 \mathrm{~kg} / \mathrm{m}^{3}$ & $1800 \mathrm{~kg} / \mathrm{m}^{3}$ \\
$\mu$ & $2 \cdot 00 \mathrm{MPa}$ & $12 \cdot 0 \mathrm{MPa}$ \\
$K$ & $3 \cdot 33 \mathrm{MPa}$ & $20 \cdot 0 \mathrm{MPa}$ \\
$\alpha$ & $428^{\mathrm{a}}$ and $228 \mathrm{kPa}^{\mathrm{b}}$ & $0 \cdot 03 \mathrm{kPa}$ \\
$\lambda$ & $0 \cdot 0$ & $153 \mathrm{kPa}$ \\
$\beta$ & $3 \cdot 48 \times 10^{-6} \mathrm{~Pa}^{-1}$ & $3 \cdot 48 \times 10^{-6} \mathrm{~Pa}^{-1}$ \\
\hline
\end{tabular}

${ }^{\mathrm{a}}$ Used in Section 4.1

${ }^{\mathrm{b}}$ Used in Section 4.3 

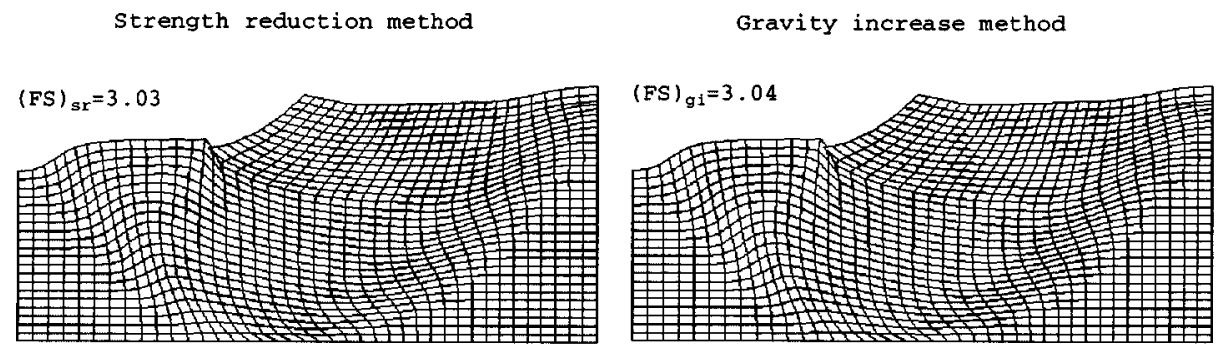

Figure 3. Deformed configurations showing computed failure mechanisms for a $30 \mathrm{~m}$ clay slope with a repose angle of $49^{\circ}$. Both the strength-reduction and gravity-increase methods of stability analysis produce mechanisms and stability factors that are virtually identical

$20 \mathrm{~m}$, and a $30^{\circ}$ angle of repose. The FEM mesh of the soil mass is constructed using 1125 bilinear quadrilateral continuum finite elements. In the gravity increase method of SSA, vertical gravity loading on the soil mass is monotonically increased until at $|\mathbf{g}|=29.626 \mathrm{~m} \mathrm{~s}^{-2}$, the slope is on the verge of instability, with the dual mechanisms of failure being those shown in Figure 3 . The computed gravitational factor of safety for this slope is $(\mathrm{FS})_{\mathrm{gi}}=3 \cdot 04$. Alternatively, in the strengthreduction method, standard gravitational loading is applied to the soil mass, and the cohesion parameter $\alpha$ is gradually reduced until the slope develops a fully initiated mechanism at $\alpha=$ $141 \mathrm{kPa}$ which provides a virtually identical computed factor of safety (FS) $)_{\mathrm{sr}}$ for the slope of 3.03. It should be noted that the Fellenius-Taylor method ${ }^{16,17}$ for this slope (with a corresponding MohrCoulomb cohesion of $302 \cdot 6 \mathrm{kPa}$ ) provides a traditional factor of safety against slope failure of $3 \cdot 17$.

Here both of the continuum/FEM methods of SSA predict a stable slope, with virtually identical computed values for factor of safety against failure, which also compare quite favourably with those computed using the Fellenius-Taylor solution. It is noted, however, that whereas for the given slope configuration the Fellenius-Taylor solution is unable to provide definite mechanism for slope failure, the proposed stability analysis techniques predict two simultaneous mechanisms, one a partially developed toe failure, and the other a more fully developed base failure. This information is very useful in a practical terms, sine it indicates which mechanisms should be considered in engineering interventions to increase the stability of the slope.

A logical question that occur in conducting slope stability analysis with the proposed continuum/FE method is how sensitive the computed factors of safety and failure mechanisms might be with respect to mesh refinement or coarsening. The same stability analysis problems were thus repeated here with FEM meshes that are twice as fine (4450 bilinear continuum elements) as the meshes shown in Figure 3. With the fine meshes (not shown here, but in Reference 1, both of the methods of SSA still produced dual failure mechanisms identical to those achieved with the mesh of Figure 3, and the computed factors of safety are virtually identical (3.02 with the fine mesh) to those computed with the coarse mesh. This simple test indicates that for the material models considered, which do not feature softening behaviours, the predicted slope stability results are convergent with mesh refinement.

\subsection{Analysis with purely frictional soils}

While the preceding examples utilized strictly cohesive, non-frictional soil behaviours, the proposed stability methods can easily be utilized with frictional soils as well. Here the stability of 

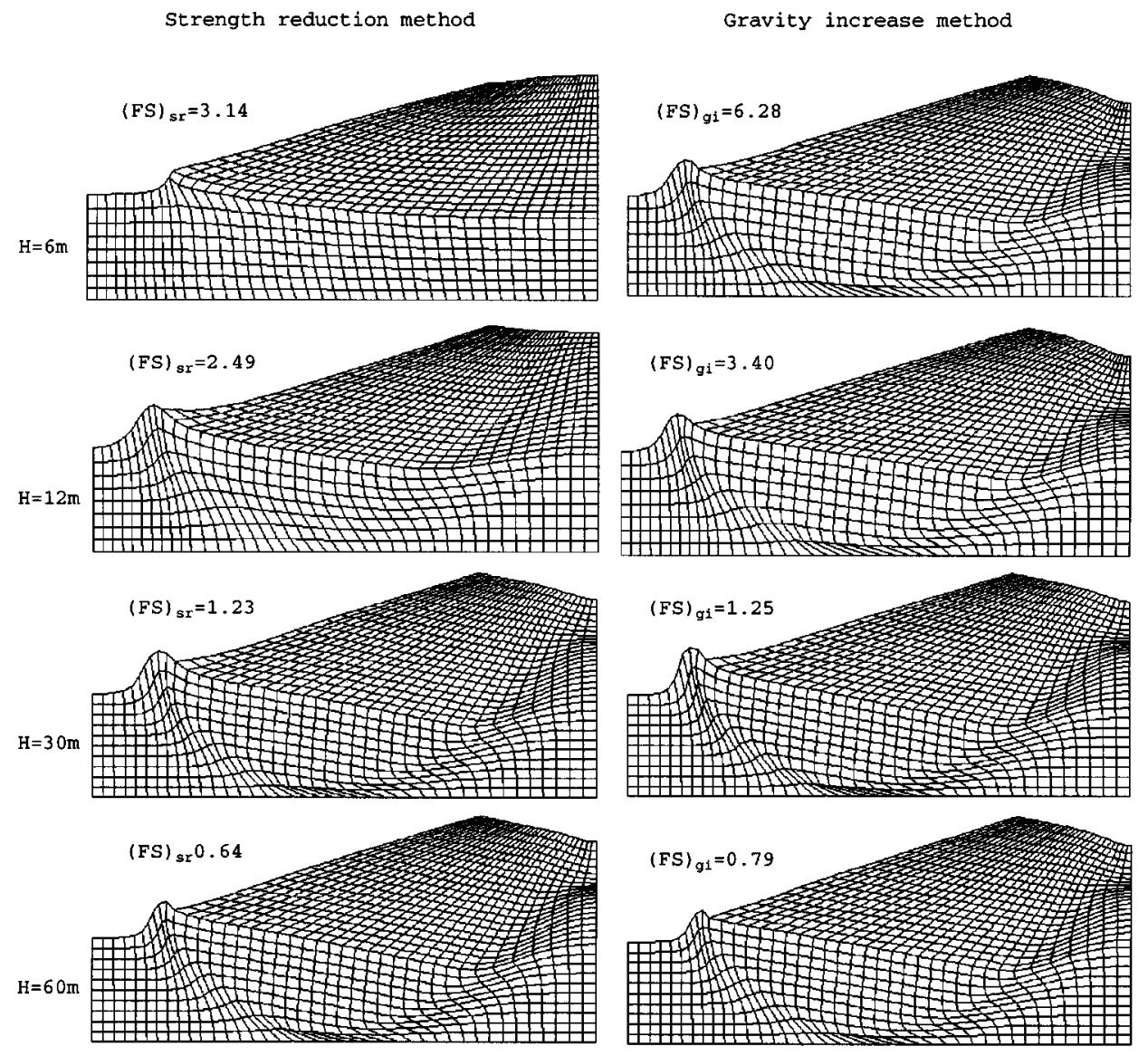

Figure 4. Limit state mechanisms and stability factors computed for a $20^{\circ}$ purely sand slope of varying heights using the strength-reducing and gravity-increase methods

a sloped sandy soil deposit having an angle or respose of $20^{\circ}$, is considered for slope heights of 6 , 12,30 , and $60 \mathrm{~m}$. The results of stability analysis for both the strength-reduction and gravityloading methods are shown in Figure 4 and indicate that for small slope heights, the strengthreduction method produces stability factors that are smaller (and thus more conservative) than the gravity-loading method of analysis. At larger slope height, both methods tend to give stability factors and failure mechanisms that are somewhat closer to each other.

For shallow slopes comprised of frictional soils as shown here, special care must be taken when using the gravity-increase method of analysis. If one were to use a traditional Mohr-Coulomb failure criterion with a sufficiently large friction angle $\phi$, or a linear Drucker-Prager failure criterion such as that of equation (14) with a sufficiently large slope $\theta$, then with increasing gravity loading, the mean normal confining stresses in the soil would increase faster than the shear stresses. Under these circumstances, the rate of strength gain in the soil under increasing gravity loading would exceed the rate of shear stress increase, and the slope would never develop a failure mechanism. In other words, for unrealistic soil models, it is possible that no limit state could exist 
for a given combination of slope angle and soil friction angle. To avoid this possibility, the gravity-based method of slope stability analysis should generally be employed with more realistic soil models (such as that in Section 3) that capture the realistic saturation of incremental frictional effects that invariably occur at high confining stresses. It should be noted that the strength reducing method of stability analysis is more robust in this regard, since it will generally yield a failure mechanism even with Mohr-Coulomb and linear Drucker-Prager failure criteria.

\subsection{Analysis with heterogeneous soil deposits}

One of the possible advantages of the proposed slope stability frame work is that is can be applied with a variety of soil conditions which can result in virtually any type of failure mechanism, and not necessarily circular arcs as are often considered in classical slope stability analysis. As an example, both the strength reduction and gravity-increase approaches are employed here to consider the stability of layered soils comprising a slope of height $30 \mathrm{~m}$ and an angle of repose of $30^{\circ}$. The computed stability factors for this slope system and the associated slope failure mechanisms are shown in Figure 5. Two points of interest can be highlighted from these results:

1. With both the strength-reduction and gravity methods of analysis, the failure mechanisms shown are quite unusual, involving both interface shear failure along the sand and clay layers, as well as a rotational failure along a circular arc.

2. The computed stability factors produced by the two methods (gravity and strength-reduction) are relatively close in value, and neither is always more or less conservative than the other.

\section{DISCUSSION AND SUMMARY}

In the slope stability analysis frameworks presented, internal soil stresses and shear strength behaviours inslopes are computed accurately using well-established non-linear incremental finite element methods which are known to converge to exact values with increasing mesh and load-step refinement. An attractive aspect of both the gravity-based framework and the strengthreduction framework is that they use virtually identical methods of analysis, and ideas to generate limit state failure mechanisms. In the gravity-based method of analysis, only a singly, simple, gravity loading parameter needs to be monotonically increased to induce failure of the slope model, whereas with the strength-reduction method only a single load-time function can be used to simultaneously reduce the shear strengths of all soils comprising a slope system. Both methods are capable of producing one or more active failure mechanisms at the computed limit state.

At present, the cost of slope stability analysis in the continuum/FEM frame work is quite reasonable and further advances in computing hardware technology should further improve the economy of the method. The representative computations shown in Section 4 each took approximately $30 \mathrm{cpu}$-min to complete on a Silicon Graphics Powerchallenge workstation using R8000 processors. ${ }^{\ddagger}$ With new generations of workstation and personal computing processors, it is

\footnotetext{
‡ The specific research subroutines and equation solvers used for these computations have not yet been optimized for performance on these workstations, and thus reductions of computing time by 50 per cent or more remain a strong possibility using the methods outlined in Reference 11.
} 

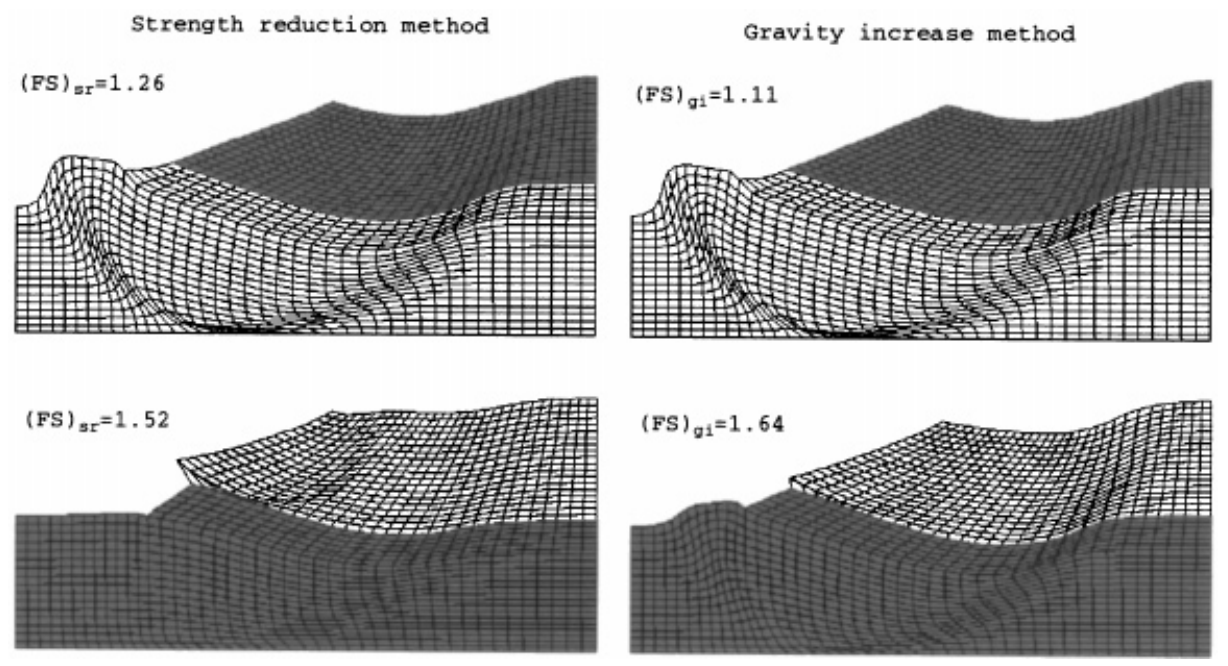

Figure 5. Deformed configurations showing computed failure mechanisms and stability factors for a layered clay/sand slope. The dark regions indicate a clay soil and the light regions a sandy soil. The slope height is $30 \mathrm{~m}$ and the angle of repose is $30^{\circ}$

reasonably expected that these analysis times will eventually fall to just a few minutes. The creation of FEM meshes for the slope models can today be created in a matter of minutes using commercially available pre- and post-processing packages. Hence both the gravity increase and strength-reduction computational approaches to slope stability analysis outlined here are competitive in terms of analysis time required with classical stability analysis methods based on slice techniques. Furthermore, the computational methods presented here are significantly more general than classical slice type methods and can account for engineering interventions such as soil nailing, retaining walls and reinforcing fabrics.

A close inspection of the computed stability factors for both the strength-reduction and gravity-increase methods of analysis indicates that the strength-reducing method more often provides stability factors that are more conservative (smaller), although, there are also instances where the gravity-increase method provides more conservative stability factors. Thus, for the total stress analysis example problems considered here, neither method demonstrated a clear superiority over the other. When the methods are applied to treat slope stability analysis problems involving seepage and/or pore pressure diffusion effects, then the methods will have certain problems where they show definite strengths. For example, the gravity-increase method of stability analysis is very well suited for analysing the stability of embankments constructed on saturated soils depots as treated in Reference 10 since the rate of construction of the embankment can be simulated with the rate at which gravity loading on the embankment is increased. And for stability analysis of earthen slopes in which active, unconfined steady-state seepage is occurring (as treated in Reference 1), the strength-reduction method of analysis is a natural choice.

While relatively simple, elastic-perfectly plastic soil models have been used in this effort, the frameworks presented are potentially amenable to usage with brittle continuum damage soil models that feature strain softening behaviours. With softening constitutive models, however, the nature and difficulty of solving the equilibrium equations becomes somewhat more complicated 
due to loss of ellipticity of the boundary value problem and the introduction of shear bands. Nevertheless, treatment of softening behaviours with these analysis frameworks is a topic worthy of exploration since such soil characteristics could well present de-stabilizing influences on earthen slopes.

\section{ACKNOWLEDGEMENTS}

This work was supported in part by an Old Gold Fellowship from the University of Iowa.

\section{REFERENCES}

1. Y.-K. Seo, 'Computational methods for elasto-plastic slope stability analysis with seepage', Ph.D dissertation, The University of Iowa, Iowa City, Iowa, USA, 1998.

2. D. Leschinsky and C.-C. Huang, 'Generalized three-dimensional slope-stability analysis', J. Geotechn. Engng, 18 (11), 1748-1764 (1992).

3. D. F. T. Nash, 'A comparative review of limit equilibrium methods of stability analysis', in M.G. Anderson and K.S. Richards (eds.), Slope Stability: Geotechnical Engineering and Geomorphology, Wiley, New York 1987, pp. 11-75.

4. J. M. Duncan, 'State of the art: limit equilibrium ad finite-element analysis of slopes', J. Geotechn. Engng, 122 (7), 577-596 (1996).

5. K. Terzaghi, 'Mechanisms of landslides', in L. Bjerrum, A. Casagrande, R. B. Peck and A. W. Skempton (eds.), From Theory to Practice in Soil Mechanics: Selections from the writings of Karl Terzaghi, Wiley, New York, 1950, pp. 202-245.

6. T.-K. Huang, 'Stability analysis of an earth dam under steady state seepage', Comput. Struct., 58 (6), 1075-1082 (1996).

7. W. F. Chen and E. Mizuno, Nonlinear Analysis in Soil Mechanics: Theory and implementation, Elsevier, Amsterdam, 1990.

8. D. V. Griffits and D. J. Kidger, 'Enhanced visualization of failure mechanisms by finite elements', Comput. Struct., 55 (2), 265-268 (1995).

9. O. C. Zienkiewicz, C. Humpheson and R. W. Lewis, 'Associated and non-associated visoplasticity and plasticity in soil mechanics', Geotechnique, 25, 671-689 (1975).

10. Y.-K. Seo and C. C. Swan, 'Stability analysis of earthen embankments on saturated soil deposits using elasto-plastic porous medium model', ASCE J. Geotechn. and Geoenv. Engng, (1999) in review.

11. C. C. Swan and I. Kosaka, 'Homogenization based analysis and design of composites', Comput. Struct., 64 (1-4), 603-621 (1997).

12. M. Gerardin and M. Hogge, 'Solving systems of nonlinear equations', H. Kardestuncer (ed.), The Finite Element Handbook, McGraw-Hill, New York, 1987.

13. J. C. Simo and R. L. Taylor, 'Consistent tangent operators for rate-independent elastoplasticity', Comput. Meth. Appl. Mech. Engng, 48, 100-118 (1985).

14. W.-F. Chen and A. F. Saleeb, Constitutive Equations for Engineering Materials, Vol. 1. Elasticity and Modeling, Wiley New York, 1982.

15. C. S. Desai, H. V. Phan, and S. Sture, 'Procedure, selection and application of plasticity models for soil', Int. J. Numer. Anal. Meth. Geomech., 4, 295-311 (1981).

16. W.Fellenius, 'Calculation of the stability of earth dams', Proceedings, Second Congress on Large Dams, Washington DC, 1936.

17. D. W. Taylor, 'Stability of earth slopes', J. Boston Soc. Civil Engineers, July issue, 1937. 